\title{
Empty sella syndrome: does it exist in children?
}

\section{Ahmed Ammar, M.B., Ch.B., D.M.Sc., Ali Al-Sultan, M.D., F.R.C.P.(C), Fatma Al Mulhim, M.B., Ch.B., and Abdulla Yousef Al Hassan, D.M.R.D.}

Departments of Neurosurgery, Internal Medicine, and Radiology, King Fahd University Hospital, Al Khobar, Saudi Arabia

The empty sella syndrome (ESS) is well documented in adults and although the same phenomenon of herniation of the arachnoid space into the enlarged sella turcica has been noted in children, it is not widely known that children suffer from this syndrome. Therefore, the aims of this paper are to increase neurosurgeons' awareness of the existence of this phenomenon in children and to add to the scant body of literature on the subject.

The authors treated 12 children, ranging in age between 2 and 8 years in whom neuroradiological studies demonstrated an enlarged sella turcica filled with cerebrospinal fluid and herniation of suprasellar and arachnoid spaces. The causes of ESS in these children were high intracranial pressure, neglected or improperly treated hydrocephalus, and suprasellar arachnoid cyst. Primary ESS was found as well. Most of the children presented with headache, abnormal body weight (the majority being underweight), and short stature. The results of hormone assays were normal in all children.

If undiagnosed and untreated, ESS in children may lead to serious consequences, including impairment of pituitary and hypothalamic function and damage to the optic chiasm. It is important to raise awareness in the neurosurgical community about the existence of ESS in children so that it can be diagnosed and treated at an early stage. A classification system for the diaphragma sellae is recapitulated.

Key Words * empty sella syndrome * hydrocephalus * tumor * intracranial pressure * children

Empty sella syndrome (ESS) is an anatomical condition in which subarachnoid space herniates into the sella, pushing the pituitary gland either to the bottom or to one side of the sella. The condition was first described in adults by Busch[4] in 1951; however, there have been few reports describing ESS in children.[1,2,5,7-13,17,22,24,25] Kaufman and colleagues[14-16] noted the relationship between high intracranial pressure (ICP) and ESS and emphasized that a prolonged increase in cerebrospinal fluid (CSF) pressure is a direct cause of ESS. They also found evidence of ESS in all of their patients with pickwickian disease.[16] Empty sella syndrome has been found in association with a constellation of conditions: diabetes insipidus, diabetes mellitus, optic atrophy, and nerve deafness, known as DIDMOAD syndrome;[20] and in Bardet-Biedl syndrome[22] and growth hormone deficiency with 18P 
monosmy[19] and dup (X) (q13.3--q21.2).[23]

\section{CLINICAL MATERIAL AND METHODS}

\section{Patient Population}

At the Department of Neurosurgery, King Fahd University Hospital, King Faisal University, Al Khobar, Saudi Arabia we treated 12 patients (seven boys and five girls) ranging in age between 2 and 8 years. Between 1989 and 1996, the patients presented with various symptoms mainly related to high ICP, short stature (10 patients), and abnormal growth rate (four patients were overweight and eight were underweight). Mental retardation was observed in four patients. None of the patients showed any sign of visual field defect; however, one patient suffered diplopia and four had marked papilledema. We believe that two of the 12 patients had primary ESS. The causes of ESS in the other 10 patients were arrested hydrocephalus (three cases) dilated third ventricle and partially functioning shunt (five cases), and suprasellar arachnoid cyst (two cases). All of these patients have been followed for periods between 26 and 54 months (mean 37 months).

\section{ILLUSTRATIVE CASES}

\section{Case 1}

This overweight 8-year-old girl presented in our clinic with mental retardation and short stature. The results of her neurological examination were normal, as were the results of endocrinological examination. Magnetic resonance (MR) imaging revealed primary ESS (Fig. 1). The patient was referred to an institute for children with developmental delays, as there was no specific treatment we could offer her.

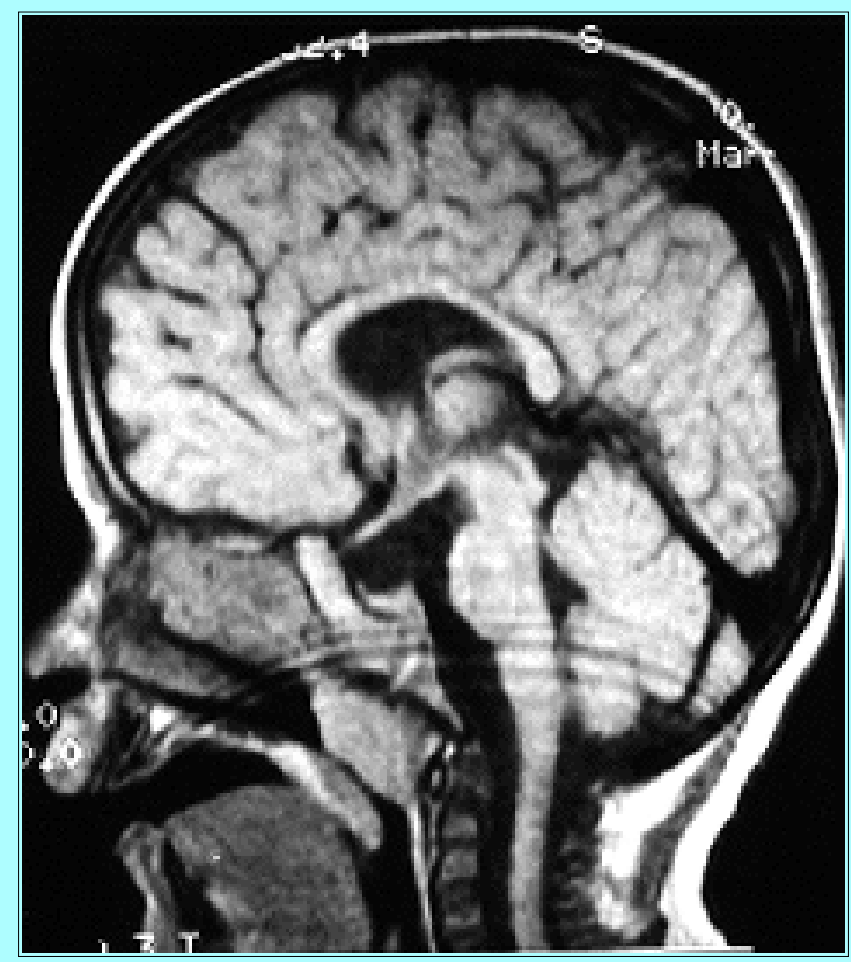

Fig. 1. Case 1. Sagittal MR image revealing a case of primary ESS in an 8-year-old girl.

\section{Case 2}


This overweight 4-year-old boy was referred to our clinic suffering from headache, unsteady gait, and short stature. He was described as irritable. Neurological examination showed the child to be fully conscious and oriented but with continuous head shaking (bubbling sign). The cranial nerves were intact, and no motor or sensory deficit was detected.

The patient's reflexes were slightly exaggerated. Funduscopic examination revealed bilateral papilledema. Magnectic resonance imaging demonstrated hydrocephalus, which was caused by a large suprasellar arachnoid cyst. The sella turcica was seen to be enlarged and the arachnoid cyst had pushed into the sella (Fig. 2). A full endocrinological examination was conducted. All hormone levels were within normal limits. The patient underwent endoscopic surgery for cyst fenestration to create drainage to the lateral and third ventricles and the suprasellar space. The patient improved remarkably postoperatively; the bubbling sign and the headache resolved after surgery. Thirteen months later, the patient was returned to our clinic again suffering from headaches. Magnectic resonance imaging revealed that the sizes of the ventricles and the cyst were unchanged. The patient underwent a second surgery for bilateral shunt placement to drain the cyst and ventricles. The patient made an excellent recovery. He has been followed for longer than 3 years, and he enjoys normal active school life.

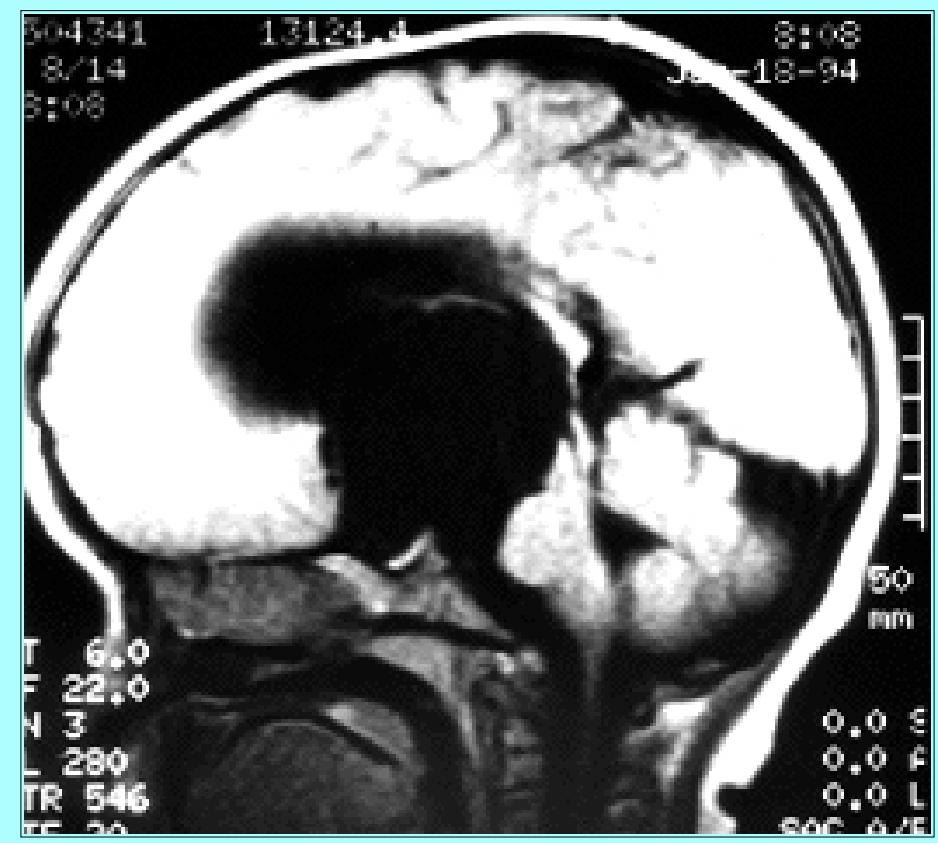

Fig. 2. Case 2. Sagittal MR image revealing a case of ESS as a consequence of longstanding high ICP due to hydrocephalus caused by a suprasellar cyst in a 4-year-old boy who presented with bubbling sign.

\section{Case 3}

This underweight 6-year-old girl was referred to our clinic with a posterior fossa tumor. The child had been treated for 3 weeks in a pediatric clinic for a case of gastroenteritis. She had been experiencing nausea, vomiting, and headache. On neurological examination, she was found to be conscious, but irritable; she had bilateral papilledema, right sixth cranial nerve palsy, diplopia, and trunk ataxia. Magnectic resonance imaging demonstrated a large midline posterior fossa tumor compressing the brainstem and the cerebeller tonsils at the level of C-1 (Arnold-Chiari malformation Type 1), as well as hydrocephalus and cervical syringomyelia; the sella turica was enlarged and filled with CSF (Fig. 3). The child underwent an emergency suboccipital craniotomy and the tumor, along with the cerebellar tonsils, was totally removed. The thinness of the bone and the presence of syringomyelia and herniation of 
suprasellar space into sella indicated that the tumor had been there for a long time.

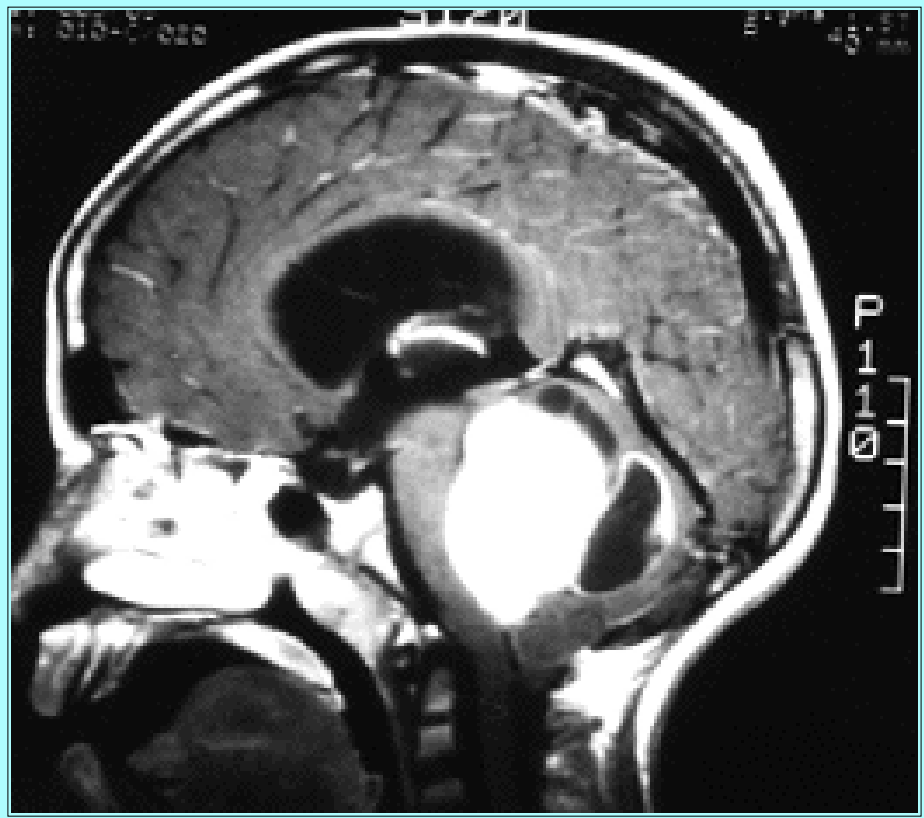

Fig. 3. Case 3. Sagittal MR image obtained in a 6-year-old girl, demonstrating a case of ESS as a complication from longstanding hydrocephalus caused by a posterior fossa astrocytoma.

Histopathological analysis revealed the tumor to be a low-grade astrocytoma. Postoperatively the patient made an excellent recovery and the results of her endocrinological examination were normal. At 2 years postsurgery she is of normal height and weight for her age. Follow-up MR imaging demonstrated that the tumor had been totally removed and that the syringomyelia had resolved; however, the sella remained enlarged.

\section{RESULTS}

The three patients with hydrocephalus and the two patients with arachnoid cysts made excellent recoveries after reduction of high ICP and cyst removal, respectively. The bubbling sign in the patient in Case 2 disappeared soon after surgery. This patient is growing normally, with normal endocrinological and neurological function. His intellectual function is age appropriate and he is doing very well in school. The patient with the posterior fossa astrocytoma has been free of neurological symptoms; and the results of all hormones study are within normal limits. The sella remained enlarged in all cases. The two patients with primary ESS showed no improvement. They remain overweight, short in stature, and intellectually developmentally delayed.

\section{DISCUSSION}

Empty sella is a term that was first introduced by Busch in 1951[4] to describe herniation of the suprasellar arachnoid space, and possibly the optic nerve, into the sella turcica because of a diaphragmatic defect. In 1926 Schaeffer[18] may have been the first to observe various anatomical forms of the diaphragma sellae; these ranged from densely thick with a complete roof to that composed of a peripheral veil. Busch, in his study of 788 sellae in patients without a history of pituitary disorders, classified the diaphragma sellae into the following categories, which were later resorted by Kaufman in 1972.[15]

Type 1-A: The diaphragma sella forms a complete seal. 
Type 1-B: A slight tunnel-shaped depression is present in the intact diaphragma sellae.

Type 2-A: An opening, 3- mm or smaller, in the diaphragma sellae exists around the hypophyseal stalk. Type 2-B: A slight funnel-shaped indentation toward the middle of the diaphragma sellae is present. Type 3-A: The diaphragma sellae is composed of a 2-mm or smaller peripheral veil, leaving the pituitary gland freely exposed and covered with arachnoid.

Type 3-B: The diaphragma sellae is as described for Type 3-A but the pituitary gland is indented, often eccentrically.

Type 3-C: The deficient diaphragma sellae is as described in Type 3-A; however, indentation of the pituitary gland is marked.

In a series of 89 autopsy studies performed by Kaufman[14] between 1966 and 1968, he found that 6 to $7 \%$ of the specimens were of the Type 3-C variety. It is important to note that these studies excluded any patient with known endocrine problems.

The relationship between prolonged high ICP, such as in cases of pseudotumor cerebri and ESS, has been studied by Kaufman[14] and Deev.[6] In these studies ESS was found in five of 40 women, in whom benign increased ICP had been diagnosed.

It is interesting that herniation of the suprasellar arachnoid space, optic nerve, optic chiasm, and sella turcica are more commonly found in cases of primary ESS than in those of secondary ESS; this is most probably because adhesions in the sella following surgery, radiation bleeding, or infection (as in cases of secondary ESS) prevent herniation. Dilation of the third ventricle or the sylvian aqueduct by hydrocephalus has also been reported in the cases of primary ESS.

Although it is generally believed that ESS is uncommon in children, there have been reports published in the last decade documenting the existence of ESS in children.[1,2,5,7-12,17,22,24,25]

In our patients, ESS was clearly evident and caused by prolonged high ICP such as that precipitated by a suprasellar arachnoid cyst or a posterior fossa tumor or that resulting from hydrocephalus or dilation of the third ventricle

It is understandable that increases in ICP and cerebrospinal fluid (CSF) cause dilation of the third ventricle. Alteration of the suprasellar CSF dynamic because of dilated recesses or the existence of a suprasellar arachnoid cyst may cause the arachnoid space, and perhaps the visual system of the third ventricle, to herniate into the sella, causing some erosion of the floor.

It would be logical to expect some sort of hormonal deficiency associated with ESS, as has been reported.[1,2,5,8,10,12,13,17,19-21,23-25] Growth hormone deficiency has repeatedly been reported as a result of ESS in children; however, in our patients and in other reports as well, the results of hormonal studies were normal.[3,7,9]

There are different methods to approach the treatment of this syndrome in adults, such as repairing the sella or performing chiasmapexy,[16] or doing nothing.[3] There is no clear treatment paradigm for children. The management strategy for children should be tailored for each patient and be based on the cause and pathophysiology of their ESS. 
Three guidelines should be considered: 1) early detection; 2) control and reduction of high ICP; and 3) hormone replacement therapy when it is needed.

Accordingly, we advocate treating these patients as soon as possible, by endoscopic third ventriculostomy and fenestration of the suprasellar arachnoid cyst, by shunting, or by early removal of the tumor. Our experience demonstrates that it is possible to ameliorate the symptoms related to high ICP or to tumor. However, the radiological signs of ESS have not changed. Early detection and treatment may prevent the development of this phenomenon.

\section{CONCLUSIONS}

Primary ESS does exist in children; it may be the consequence of a congenital defect in the diaphragma sellae or the product of prolonged high ICP or alteration of suprasellar CSF pulsation. This syndrome, if neglected, may cause visual field defects and hormonal disorders; therefore, every effort should be made to correct this condition in children. Neurosurgeons should consider the following possible pathophysiological mechanisms of ESS in children:

1) Enlargement of the ventricles and increased ICP leads to increased head circumference and thinning of the sellar floor, increasing its capacity.

2) The pulsatile high pressure of the third ventricle leads to widening of the diaphragmatic opening and forces the suprasellar cistern into the sella.

3) The direct pulsatile pressure on the pituitary gland may lead to atrophy of the gland and alteration of its function.

\section{Acknowledgment}

The authors thank Dr. Bruce A. Kaufman, University of Washington, for sending us copies of the original work of his father Dr. Benjamin Kaufman.

\section{References}

1. Akcurin S, Ocal G, Berberoglu M, et al: Association of empty sella and neuroendocrine disorders in childhood. Acta Paediatr Jpn 37:347-351, 1995

2. Anton Botella F, Labarga Echeverria P, Miranda JJ: [Primary empty sella turcica with partial hypopituitarism and diagnosis with magnetic resonance.] An Med Interna 7:654-655, 1990 (Letter) (Sp)

3. Braatvedt GD, Corrall RJ: The empty sella syndrome: much ado about nothing. Br J Hosp Med 47:523-525, 1992

4. Busch W: Die Morphologie der Sella turcica und ihre Beziehungen zur Hypophyse. Virchows Arch A Pathol Pathol Anat 320:437-458, 1951

5. Cacciari E, Zucchini S, Ambrosetto P, et al: Empty sella in children and adolescents with possible hypothalamic-pituitary disorders. J Clin Endocrinol Metab 78:767-771, 1994

6. Deev AS: "Empty" sella turcica in benign intracranial hypertension. Zh Nevropatol Psikhiatr 91:106-108, 1991 
7. Dotsch J, Blum WF, Rascher W, et al: Short stature and low IGF-1 and IGFBP-3 despite normal growth hormone secretion in a 4 year-old girl with primary empty sella syndrome. J Pediatr Endocrinol Metab 9:415-418, 1996

8. Estopinan V, Fortea L, Gracia P, et al: [Primary empty sella turcica: clinical aspects and hormonal study of 15 cases.] An Med Interna 8:537-541, 1991 (Sp)

9. Foresti M, Guidali A, Susanna P: [Primary empty sella. Incidence in 500 asymptomatic subjects examined with magnetic resonance.] Radiol Med 81:803-807, 1991 (Ita)

10. Gallardo E, Schachter D, Caceres E, et al: The empty sella: results of treatment in 76 successive cases and high frequency of endocrine and neurological disturbances. Clin Endocrinol 37:529-533, 1992

11. Gonianakis M, Segas J, Kontou-Fili K, et al: Empty sella syndrome, diagnosed as allergic rhinitis. ORL J Otorhinolaryngol Relat Spec 54:91-94, 1992

12. Hung W, Fitz CR: The primary empty-sella syndrome and diabetes insipidus in a child. Acta Paediatr 81:459-461, 1992

13. Jordan RM, Kendall JW, Kerber CW: Primary empty sella syndrome analysis of the clinical characteristics, radiographic features, pituitary function and cerebrospinal fluid adenohypophysial hormone. Am J Med 62:569-580, 1977

14. Kaufman B: The "empty" sella turcica--a manifestation of the intrasellar subarachnoid space. Radiology 90:931-941, 1968

15. Kaufman B, Chamberlin WB Jr: The ubiquitous "empty" sella turcica. Acta Radiol 13:413-425, 1972

16. Kaufman B, Tomsak RL, Kaufman BA, et al: Herniation of the suprasellar visual system and the third ventricle into empty sellae: morphologic and clinical considerations. AJR 152:597-608, 1989

17. Rapaport R, Logrono R: Primary empty sella syndrome in childhood: association with precocious puberty. Clin Pediatr 30:466-471, 1991

18. Schaeffer JP: Some points in the regional anatomy of the optic pathway with special reference to tumors of the hypophysis cerebri and resulting ocular changes. Anat Rec 28:243-279, 1924

19. Schober E, Scheibenreiter S, Frisch H: 18P monosomy with GH-deficiency and empty sella: good response to GH-treatment. Clin Genet 47:254-256, 1995

20. Soliman AT, Bappal B, Darwish A, et al: Growth hormone deficiency and empty sella in DIDMOAD syndrome: an endocrine study. Arch Dis Child 73:251-253, 1995

21. Soliman AT, Rajab A, AlSalmi I, et al: Empty sella, impaired testosterone secretion, and defective hypothalamic-pituitary growth and gonadal axes in children with Bardet-Biedl syndrome. Metabolism 45:1230-1234, 1996

22. Unsinn K, Glatzl J: [Empty sella syndrome in childhood.] Pediatr Padol 27:39-41, 1991 (Ger)

23. Yokoyama Y, Narahara K, Tsuji K, et al: [Growth hormone deficiency and empty sella syndrome in a boy with $\operatorname{dup}(X)$ (q13.3----q21.2).] Am J Med Genet 42:660-664, 1992 (Ita) 
24. Zamboni G, Ziviani L, Antoniazzi F, et al: Empty sella syndrome: 2 cases to show its polymorphism. Pediatr Med Chir 19:65-67, 1997

25. Zucchini S, Ambrosetto P, Carla G, et al: Primary empty sella syndrome: differences and similarities between children and adults. Acta Paediatr 84:1382-1385, 1995

Manuscript received March 2, 1999.

Accepted in final form July 17, 1999.

Address reprint requests to: Prof. A. Ammar, King Fahd University Hospital, P.O. Box 40040, Al Khobar, Saudi Arabia 31952. 